Mathematical Modelling and AnAlysis

Volume 21 Number 2, March 2016, 270-281

http://dx.doi.org/10.3846/13926292.2016.1154113

(C) Vilnius Gediminas Technical University, 2016
Publisher: Taylor\&Francis and VGTU

http://www.tandfonline.com/TMMA

ISSN: $1392-6292$

eISSN: $1648-3510$

\title{
On Three-Point Boundary Value Problem
}

\section{Nadezhda Sveikate ${ }^{a}$}

\author{
${ }^{a}$ Daugavpils University \\ Parades street 1, Daugavpils, LV-5401 \\ E-mail: nsveikate@inbox.lv
}

Received September 8, 2015; revised February 9, 2016; published online March 15, 2016

\begin{abstract}
Three-point boundary value problems for the second order nonlinear ordinary differential equations are considered. Existence of solutions are established by using the quasilinearization approach. As an application, the Emden-Fowler type problems with nonresonant and resonant linear parts are considered to demonstrate our results.
\end{abstract}

Keywords: nonlinear three-point boundary value problem, resonant problem, quasilinearization process.

AMS Subject Classification: 34B15; 34B10.

\section{Introduction}

We consider the nonlinear second order differential equation

$$
x^{\prime \prime}+k^{2} x=f\left(t, x, x^{\prime}\right)
$$

with three-point boundary conditions

$$
x(0)=0, \quad x(1)=\alpha x(\eta),
$$

where $0<\eta<1, \alpha>0, f$ may be unbounded.

The three-point boundary value problem belongs to the so-called "nonlocal" or "multi-point" or "m-point" boundary value problem (BVP in short)

$$
\begin{aligned}
& x^{\prime \prime}=f\left(t, x, x^{\prime}\right), \\
& x(0)=0, \quad x(1)=\sum_{i=1}^{m-2} a_{i} x\left(\xi_{i}\right),
\end{aligned}
$$

where $f:[0,1] \times R^{2} \rightarrow R$ is a continuous function, $a_{i} \in R, \xi_{i}(0,1), i=$ $1,2, \ldots, m-2,0<\xi_{1}<\xi_{2}<\cdots<\xi_{m-2}<1$.

The nonlocal BVPs for ordinary differential equations arise in a variety of different areas of applied mathematics and physics, and describe many phenomena in the applied mathematical sciences. For example, the vibrations of 
a guy wire of a uniform cross-section and composed on $\mathrm{N}$ parts of different densities can be set up as a multi-point BVP (see [13]).

The study of multi-point BVP for linear second-order ordinary differential equations was initiated by Il'in and Moiseev in [7], [8].

Since then, by applying the Leray-Schauder continuation theorem, nonlinear alternative of Leray-Schauder, or coincidence degree theory, many authors studied more general nonlinear multi-point BVPs, for example, Gupta [3], [5], Gupta, Ntouyas and Tsamatos [6], Feng and Webb [2], [1], Ma [10], [12], Ntouyas [14], Webb [23].

The existence problems of positive solution of the nonlocal boundary value problem were studied by Ma [11], Webb and Infante [24], [25], Liu [9], Sun and Liu [17].

We are interested in the BVP (1.1), (1.2) at resonance. This problem was studied by Feng and Webb [2], Gupta [4], Ma [12], Przeradzki and Sta'nczy [15]. The most of authors have proved the existence of solutions of (1.1), (1.2), assuming that nonlinearity $f$ is sublinear with respect to the second and third variables. Our main result in this paper is to get conditions which guarantee that the resonant BVP is solvable. To get the existence of a solution to the resonant BVP (1.1), (1.2), we use the quasilinearization process [26], [27], which consists in reducing the resonant BVP to nonresonant one using suitable estimations of expected solutions.

The paper consists of the introduction, 4 sections, the conclusions and references.

\section{Definition}

We consider the BVP (1.1), (1.2).

Definition 1. If the homogeneous BVP

$$
\begin{aligned}
& \left(l_{2} x\right)(t)=0, \\
& x(0)=0, \quad x(1)-\alpha x(\eta)=0
\end{aligned}
$$

has only the trivial solution, then the BVP (1.1), (1.2) with a linear part $\left(l_{2} x\right)(t):=x^{\prime \prime}+k^{2} x$ is called nonresonant. Otherwise, if the homogeneous $B V P$ (2.1), (2.2) has a nontrivial solution, then the BVP (1.1), (1.2) with a linear part $\left(l_{2} x\right)(t)$ is called resonant.

For example, BVP

$$
x^{\prime \prime}=0, \quad x(0)=0, \quad x(1)=x(1 / 2)
$$

is nonresonant, but BVP

$$
x^{\prime \prime}=0, \quad x(0)=0, \quad x(1)=2 x(1 / 2)
$$

is resonant, that is, it has nontrivial solution $x(t)=C t, \forall C$.

The BVP

$$
x^{\prime \prime}+k^{2} x=0, \quad x(0)=0, \quad x(1)=x(1 / 2)
$$


is nonresonant if $\sin k \neq \sin k / 2$; the coefficient $k$ belongs to one of the intervals

$$
\left[0, \frac{2 \pi}{3}\right),\left(\frac{2 \pi}{3}, 2 \pi\right),\left(2 \pi, \frac{10 \pi}{3}\right),\left(\frac{10 \pi}{3}, 4 \pi\right),\left(4 \pi, \frac{14 \pi}{3}\right), \ldots
$$

These intervals are called nonresonant intervals.

\section{Quasilinear problem}

We consider a quasilinear BVP

$$
\begin{aligned}
& x^{\prime \prime}+k^{2} x=\varphi(t, x), \\
& x(0)=0, \quad x(1)-\alpha x(\eta)=0 .
\end{aligned}
$$

If $\varphi$ is continuous and bounded with $M$ in modulus and corresponding homogeneous differential equation

$$
x^{\prime \prime}+k^{2} x=\varphi(t, x),
$$

with boundary conditions (3.2) has only the trivial solution, then the BVP (3.1), (3.2) is solvable in accordance with the Theorem of existence at [21].

The solution of the quasilinear BVP (3.1), (3.2) can be written in the integral form

$$
x(t)=\int_{0}^{1} G(t, s) \varphi(s, x(s)) d s,
$$

where $G(t, s)$ is the Green's function for the respective homogeneous BVP (3.3), (3.2). If $|\varphi(t, x)| \leq M$, then from (3.4) it follows that

$$
|x(t)| \leq \Gamma M,
$$

where $\Gamma$ is bound for $|G(t, s)|$.

For the linear part $\left(l_{2} x\right)(t)=x^{\prime \prime}+k^{2} x$, where $\sin k-\alpha \sin k \eta \neq 0$, with given boundary conditions, the Green's function [16], [22] is given by

$$
G(t, s)= \begin{cases}\frac{(\sin k(1-s)-\alpha \sin k(\eta-s)) \sin k t}{k(\alpha \sin k \eta-\sin k)}, & 0 \leq t \leq s \leq \eta, \\ \frac{\sin k s(\sin k(1-t)-\alpha \sin k(\eta-t))}{k(\alpha \sin k \eta-\sin k)}, & s \leq t, s \leq \eta, \\ \frac{\sin k(1-s) \sin k t}{k(\alpha \sin k \eta-\sin k)}, & t \leq s, \eta \leq s, \\ \frac{\alpha \sin k \eta \sin k(t-s)+\sin k s \sin k(1-t)}{k(\alpha \sin k \eta-\sin k)}, & \eta \leq s \leq t \leq 1 .\end{cases}
$$

The Green's function (3) is bounded by $|G(t, s)| \leq \Gamma$. Then it follows from the integral form (3.4) that a solution $x(t)$ satisfies the inequality

$$
|x(t)| \leq \Gamma M .
$$




\section{Quasilinearization process}

We consider the BVP

$$
\begin{aligned}
& x^{\prime \prime}+k^{2} x=f(t, x), \\
& x(0)=0, \quad x(1)-\alpha x(\eta)=0,
\end{aligned}
$$

where $f(t, x)$ is a continuous function.

We use a quasilinearization approach. Previously this approach was used for the study of different BVPs in $[18,19,20]$.

1. First we modify the equation by adding a linear part so that the resulting linear part is not resonant

$$
x^{\prime \prime}+k^{2} x+\varepsilon^{2} x=\varepsilon^{2} x+f(t, x)=: F(t, x),
$$

where $\sin \sqrt{k^{2}+\varepsilon^{2}}-\alpha \sin \eta \sqrt{k^{2}+\varepsilon^{2}} \neq 0$.

2. We choose a constant $N>0$ and truncate the right hand side

$$
x^{\prime \prime}+\left(k^{2}+\varepsilon^{2}\right) x=F_{N}(t, x):=F(t, \delta(-N, x, N)),
$$

where

$$
\delta(-N, x, N)=\left\{\begin{array}{cl}
N, & x>N \\
x, & -N \leq x \leq N \\
-N, & x<-N .
\end{array}\right.
$$

3. We check the inequality $\Gamma M \leq N$, where $\Gamma$ is the estimate of the Green's function associated with the linear part in (4.4), $M=\sup _{I \times \mathbb{R}}\left|F_{N}(t, x)\right|$.

Therefore, the solution of the quasilinear BVP (4.4), (4.2) is also a solution of the resonant BVP (4.1), (4.2) with unbounded nonlinearity. We can say that the boundary value problem (4.1), (4.2) allows for quasilinearization with respect to the linear part $\left(L_{2} x\right)(t):=x^{\prime \prime}+\left(k^{2}+\varepsilon^{2}\right) x$.

It appears that the following theorem is valid.

Theorem 1. Suppose that for the modified quasilinear BVP

$$
x^{\prime \prime}+k^{2} x+\varepsilon^{2} x=F_{N}(t, x), \quad x(0)=0, \quad x(1)=\alpha x(\eta),
$$

where $F_{N}(t, x)=k^{2} \delta(-N, x, N)+f(t, \delta(-N, x, N)), \delta$ is truncation function, $N>0$ and the linear part is no more resonant with respect to the given boundary conditions, the inequality

$$
\Gamma M \leq N
$$

holds, where $\Gamma$ is the estimate for the Green's function associated with the linear part in (4.5) and $M=\sup _{I \times R^{2}}\left|F_{N}(t, x)\right|$.

Then the problem (4.1), (4.2) has a solution such that $|x(t)| \leq N \forall t \in[0,1]$.

Proof. The proof follows from the above arguments. We apply (4.3) and (4.4). By Theorem of existence [21] the quasilinear BVP (4.4), (4.2) is solvable and has a solution $x(t)$, which can be written in the integral form (3.4) using Green's function. 
The inequality (4.6) is fulfilled, therefore

$$
|x(t)| \leq N \quad \text { for all } t \in[0,1] .
$$

For these values of $x$ the original equation (4.1) and the modified equation (4.4) are equivalent. It follows that $x(t)$ is also a solution of the original BVP (4.1), (4.2).

\section{Application}

Consider the Emden-Fowler type BVP

$$
\begin{aligned}
& x^{\prime \prime}+k^{2} x=-\lambda|x|^{p} \operatorname{sgn} x, \quad p>0, \quad p \neq 1, \quad \lambda>0, \\
& x(0)=0, \quad x(1)=x(1 / 2) .
\end{aligned}
$$

The BVP is resonant if $\sin k-\sin k / 2=0$. That is, we give two series of eigenvalues

$$
k=4 \pi(n+1), \quad k=\frac{2 \pi}{3}+\frac{4 \pi n}{3}, \quad n=0,1,2, \ldots
$$

that are combined and form the nonresonant intervals

$$
\left[0, \frac{2 \pi}{3}\right),\left(\frac{2 \pi}{3}, 2 \pi\right),\left(2 \pi, \frac{10 \pi}{3}\right),\left(\frac{10 \pi}{3}, 4 \pi\right),\left(4 \pi, \frac{14 \pi}{3}\right), \ldots
$$

\subsection{Nonresonant case}

We consider the BVP

$$
\begin{aligned}
& x^{\prime \prime}=-|x|^{p} \operatorname{sign} x, \quad p>0, \quad p \neq 1, \\
& x(0)=0, \quad x(1)=x(1 / 2) .
\end{aligned}
$$

The linear part is nonresonant with respect to given boundary conditions, function $-|x|^{p} \operatorname{sign} x$ is unbounded. Theorem 1 was applied modifying the equation as

$$
x^{\prime \prime}+\varepsilon^{2} x=\varepsilon^{2} x-|x|^{p} \operatorname{sign} x,
$$

where the linear part $\left(L_{2} x\right)(t):=x^{\prime \prime}+\varepsilon^{2} x$ is nonresonant with respect to the boundary conditions (5.3), if $\sin \varepsilon-\sin \varepsilon / 2 \neq 0$.

The results of successful quasilinearizations are summarized in the Table 1.

Example 1. We consider the nonresonant BVP

$$
x^{\prime \prime}=-|x|^{\frac{16}{15}} \operatorname{sign} x, x(0)=0, x(1)=x\left(\frac{1}{2}\right) .
$$

We apply quasilinearization process and rewrite (5.4) equivalently

$$
x^{\prime \prime}+\frac{\pi^{2}}{9} x=\frac{\pi^{2}}{9} x-|x|^{\frac{16}{15}} \operatorname{sgn} x .
$$


Table 1. Results of calculations for the nonresonant BVP (5.2), (5.3).

\begin{tabular}{|c|c|c|c|c|}
\hline$p$ & nonresonant intervals & $\varepsilon$ & $\Gamma_{\varepsilon} M_{\varepsilon}$ & $N_{\varepsilon}$ \\
\hline 2 & $\left(\begin{array}{l}\left.0 ; \frac{2 \pi}{3}\right) \\
\left.\frac{2 \pi}{3} ; 2 \pi\right)\end{array}\right.$ & $\begin{array}{l}\frac{\pi}{3} \\
\frac{4 \pi}{3}\end{array}$ & $\begin{array}{l}1.177 \\
19.795\end{array}$ & $\begin{array}{l}1.324 \\
21.179\end{array}$ \\
\hline$\frac{6}{5}$ & 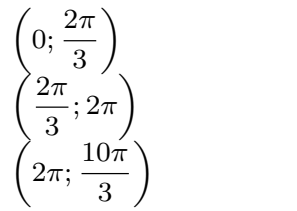 & $\begin{array}{l}\frac{\pi}{3} \\
\frac{4 \pi}{3} \\
\frac{8 \pi}{3}\end{array}$ & $\begin{array}{l}0.456 \\
5.027 \cdot 10^{5} \\
1.029 \cdot 10^{9}\end{array}$ & $\begin{array}{l}2.043 \\
21.426 \cdot 10^{5} \\
2.194 \cdot 10^{9}\end{array}$ \\
\hline$\frac{16}{15}$ & $\left.\begin{array}{l}\left(0 ; \frac{2 \pi}{3}\right) \\
\left.\frac{2 \pi}{3} ; 2 \pi\right) \\
2 \pi ; \frac{10 \pi}{3} \\
\left(\frac{10 \pi}{3} ; 4 \pi\right.\end{array}\right)$ & $\begin{array}{l}\frac{\pi}{3} \\
\frac{4 \pi}{3} \\
\frac{8 \pi}{3} \\
\frac{11 \pi}{3}\end{array}$ & $\begin{array}{l}0.406 \\
4.927 \cdot 10^{17} \\
1.058 \cdot 10^{27} \\
7.785 \cdot 10^{31}\end{array}$ & $\begin{array}{l}5.223 \\
60.215 \cdot 10^{17} \\
6.466 \cdot 10^{27} \\
9.114 \cdot 10^{31}\end{array}$ \\
\hline$\frac{15}{16}$ & $\begin{array}{l}\left(0 ; \frac{2 \pi}{3}\right) \\
\left(\frac{2 \pi}{3} ; 2 \pi\right) \\
\left(2 \pi ; \frac{10 \pi}{3}\right) \\
\left(\frac{10 \pi}{3} ; 4 \pi\right)\end{array}$ & $\begin{array}{l}\frac{\pi}{3} \\
\frac{4 \pi}{3} \\
\frac{8 \pi}{3} \\
\frac{11 \pi}{3}\end{array}$ & $\begin{array}{l}0.023 \\
1.328 \cdot 10^{-21} \\
6.182 \cdot 10^{-31} \\
1.213 \cdot 10^{-34}\end{array}$ & $\begin{array}{l}0.305 \\
16.520 \cdot 10^{-21} \\
38.464 \cdot 10^{-31} \\
1.443 \cdot 10^{-34}\end{array}$ \\
\hline$\frac{5}{6}$ & 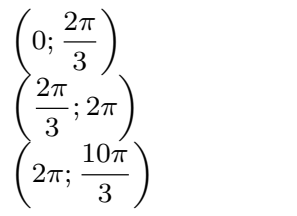 & $\begin{array}{l}\frac{\pi}{3} \\
\frac{4 \pi}{3} \\
\frac{8 \pi}{3}\end{array}$ & $\begin{array}{l}0.165 \\
1.036 \cdot 10^{-8} \\
5.058 \cdot 10^{-12}\end{array}$ & $\begin{array}{l}0.779 \\
4.645 \cdot 10^{-8} \\
39.333 \cdot 10^{-12}\end{array}$ \\
\hline$\frac{1}{2}$ & $\left(\begin{array}{l}\left.0 ; \frac{2 \pi}{3}\right) \\
\left.\frac{2 \pi}{3} ; 2 \pi\right)\end{array}\right.$ & $\begin{array}{l}\frac{\pi}{3} \\
\frac{4 \pi}{3}\end{array}$ & $\begin{array}{l}0.892 \\
3.665 \cdot 10^{-3}\end{array}$ & $\begin{array}{l}1.3212 \\
4.733 \cdot 10^{-3}\end{array}$ \\
\hline
\end{tabular}

The linear part in (5.5) is no resonant with respect to the given three-point boundary conditions. The function

$$
f(x):=\frac{\pi^{2}}{9} x-|x|^{\frac{16}{15}} \operatorname{sgn} x
$$

is continuous, odd and has a local maximum at $x_{0} \approx 1.515$. We truncate it. Define

$$
M:=f\left(x_{0}\right)=f(1.515)=0.104 .
$$

Solve the equation $f(x)=-M$ for $x>0$. The solution is $N:=5.223$. 
Define the truncated function

$$
F_{N}(x)=\left\{\begin{array}{rc}
-0.104, & x>5.223 \\
\frac{\pi^{2}}{9} x-|x|^{\frac{16}{15}} \operatorname{sgn} x, & -5.223 \leq x \leq 5.223, \\
0.104, & x<-5.223 .
\end{array}\right.
$$

The function $F_{N}(x)$ is continuous and bounded by the number $M$. Therefore the quasilinear BVP

$$
x^{\prime \prime}+\pi^{2} x / 9=F_{N}(x), \quad x(0)=0, \quad x(1)=x(1 / 2)
$$

has a solution $x(t)$. A solution $x(t)$ satisfies the integral form

$$
x(t)=\int_{0}^{1} G(t, s) F_{N}(x(s)) d s,
$$

where $G$ is the Green's function for the respective homogeneous BVP

$$
x^{\prime \prime}+\pi^{2} x / 9=0, \quad x(0)=0, \quad x(1)=x(1 / 2) .
$$

It follows from integral form (5.8) that solution $x(t)$ satisfies inequality

$$
|x(t)| \leq \Gamma M \quad \forall t \in[0,1]
$$

where $\Gamma=9(1+\sqrt{3}) /(2 \pi)$ is the estimate of the Green's function. Since

$$
\Gamma M=\frac{9(1+\sqrt{3})}{2 \pi} 0.104 \approx 0.406<5.223 \approx N,
$$

then from (5.9) it follows that solution $x(t)$ satisfies inequality

$$
|x(t)| \leq N \quad \forall t \in[0,1] .
$$

This means that the BVP (5.4) allows for quasilinearization for $\varepsilon=\pi / 3$ from first nonresonance interval $(0 ; 2 \pi / 3)$, and the solution $x(t)$ of the quasilinear BVP (5.7) is also a solution of the original resonant BVP (5.4).

The BVP (5.4) allows for quasilinearization also for $\varepsilon=4 \pi / 3$ from second nonresonance interval $(2 \pi / 3 ; 2 \pi)$, for $\varepsilon=8 \pi / 3$ from third nonresonance interval $(2 \pi ; 10 \pi / 3)$ and for $\varepsilon=11 \pi / 3$ from fourth nonresonance interval $(10 \pi / 3 ; 4 \pi)$.

Applying the shooting method we find different solutions for each quasilinearization. For results, see Figure 1.

\subsection{Resonant case}

We consider the resonant BVP

$$
\begin{aligned}
& x^{\prime \prime}+\frac{4 \pi^{2}}{9} x=-|x|^{p} \operatorname{sign} x, \quad p>0, \quad p \neq 1, \\
& x(0)=0, \quad x(1)=x(1 / 2) .
\end{aligned}
$$




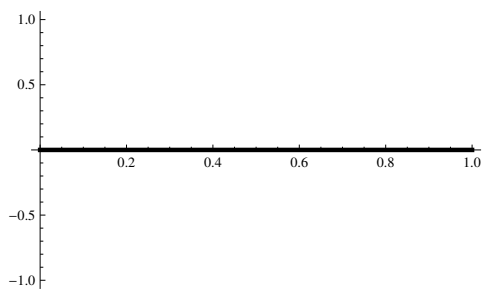

(a) The trivial solution of the BVP (5.4). Solution related to first quasilinearization with linear part $\left(L_{2} x\right)(t):=x^{\prime \prime}+\frac{\pi^{2}}{9} x$ of $(5.4)$.

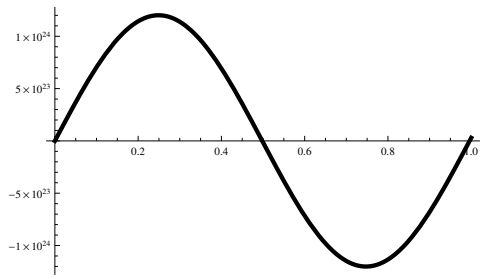

(c) Solution of the BVP (5.4) given for the initial data $x(0)=0, x^{\prime}(0)=$ $7.5 \cdot 10^{24}$. Solution related to third quasilinearization with linear part $\left(L_{2} x\right)(t):=x^{\prime \prime}+\frac{8 \pi^{2}}{9} x$ of $(5.4)$.

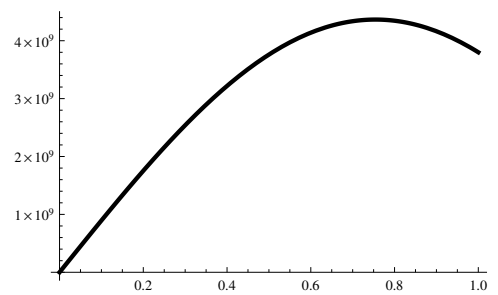

(b) Solution of the BVP (5.4) given for the initial data $x(0)=0, x^{\prime}(0)=$ $9 \cdot 10^{9}$. Solution related to second quasilinearization with linear part $\left(L_{2} x\right)(t):=x^{\prime \prime}+\frac{4 \pi^{2}}{9} x$ of $(5.4)$.

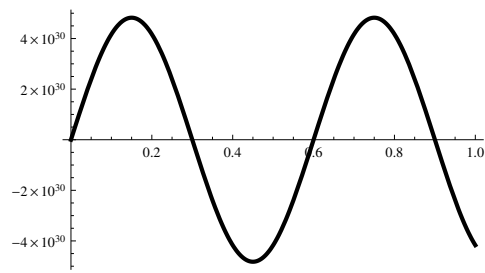

(d) Solution of the BVP (5.4) given for the initial data $x(0)=0, x^{\prime}(0)=$ $5 \cdot 10^{31}$. Solution related to fourth quasilinearization with linear part $\left(L_{2} x\right)(t):=x^{\prime \prime}+\frac{11 \pi^{2} x}{9}$ of $(5.4)$.

Figure 1. Different solutions of the BVP (5.4).

The linear part $\left(l_{2} x\right)(t):=x^{\prime \prime}+\frac{4 \pi^{2}}{9} x$ is resonant with respect to the given boundary conditions.

Theorem 1 was applied multiply modifying the equation as

$$
x^{\prime \prime}+\frac{4 \pi^{2}}{9} x+\varepsilon^{2} x=\varepsilon^{2} x-|x|^{p} \operatorname{sign} x,
$$

where the linear part $\left(L_{2} x\right)(t):=x^{\prime \prime}+\left(4 \pi^{2} / 9+\varepsilon^{2}\right) x$ is nonresonant with respect to the boundary conditions (5.12), if

$$
\sin \sqrt{4 \pi^{2} / 9+\varepsilon^{2}}-\sin \frac{\sqrt{4 \pi^{2} / 9+\varepsilon^{2}}}{2} \neq 0 .
$$

We choose $\varepsilon$ so that the resulting coefficient $\sqrt{4 \pi^{2} / 9+\varepsilon^{2}}$ of the modified linear part $\left(L_{2} x\right)(t)$ lies in the one of nonresonant intervals (5.1).

The results of successful quasilinearizations are summarized in the Table 2.

Example 2. Consider the BVP

$$
x^{\prime \prime}+\frac{4 \pi^{2}}{9} x=-|x|^{\frac{16}{15}} \operatorname{sgn} x, \quad x(0)=0, \quad x(1)=x\left(\frac{1}{2}\right) .
$$


Table 2. Results of calculations for the resonant BVP (5.11), (5.12).

\begin{tabular}{|c|c|c|c|c|}
\hline$p$ & nonresonance intervals & $\varepsilon$ & $\Gamma_{\varepsilon} M_{\varepsilon}$ & $N_{\varepsilon}$ \\
\hline$\frac{3}{2}$ & $\left(\frac{2 \pi}{3}, 2 \pi\right)$ & $\pi$ & 47.687 & 121.848 \\
\hline$\frac{5}{4}$ & $\begin{array}{l}\left(\frac{2 \pi}{3}, 2 \pi\right) \\
\left(2 \pi, \frac{10 \pi}{3}\right.\end{array}$ & $\begin{array}{l}\pi \\
\frac{8 \pi}{3}\end{array}$ & $\begin{array}{l}1.301 \cdot 10^{3} \\
1.355 \cdot 10^{6}\end{array}$ & $\begin{array}{l}10.931 \cdot 10^{3} \\
2.482 \cdot 10^{7}\end{array}$ \\
\hline$\frac{16}{15}$ & $\begin{array}{l}\left(\frac{2 \pi}{3}, 2 \pi\right) \\
\left(2 \pi, \frac{10 \pi}{3}\right) \\
\left(\frac{10 \pi}{3}, 4 \pi\right)\end{array}$ & $\begin{array}{c}\pi \\
\frac{8 \pi}{3} \\
\frac{11 \pi}{3}\end{array}$ & $\begin{array}{l}6.442 \cdot 10^{13} \\
1.122 \cdot 10^{27} \\
7.785 \cdot 10^{31}\end{array}$ & $\begin{array}{l}1.075 \cdot 10^{15} \\
6.466 \cdot 10^{27} \\
9.114 \cdot 10^{31}\end{array}$ \\
\hline$\frac{15}{16}$ & $\begin{array}{l}\left(\frac{2 \pi}{3}, 2 \pi\right) \\
\left(\begin{array}{l}2 \pi, \frac{10 \pi}{3} \\
\frac{10 \pi}{3}, 4 \pi\end{array}\right)\end{array}$ & $\begin{array}{c}\pi \\
\frac{8 \pi}{3} \\
\frac{11 \pi}{3}\end{array}$ & $\begin{array}{l}9.677 \cdot 10^{-18} \\
6.556 \cdot 10^{-31} \\
1.211 \cdot 10^{-34}\end{array}$ & $\begin{array}{c}164.459 \cdot 10^{-18} \\
38.464 \cdot 10^{-31} \\
1.443 \cdot 10^{-34}\end{array}$ \\
\hline$\frac{4}{5}$ & $\begin{array}{l}\left(\frac{2 \pi}{3}, 2 \pi\right) \\
\left(2 \pi, \frac{10 \pi}{3}\right)\end{array}$ & $\begin{array}{l}\pi \\
\frac{8 \pi}{3}\end{array}$ & $\begin{array}{l}2.891 \cdot 10^{-6} \\
4.604 \cdot 10^{-10}\end{array}$ & $\begin{array}{l}14.557 \cdot 10^{-6} \\
8.005 \cdot 10^{-10}\end{array}$ \\
\hline$\frac{2}{3}$ & $\left(\frac{2 \pi}{3}, 2 \pi\right)$ & $\pi$ & $5.092 \cdot 10^{-4}$ & $14.552 \cdot 10^{-4}$ \\
\hline
\end{tabular}

Using the quasilinearization process we obtain the quasilinear equation

$$
x^{\prime \prime}+\left(4 \pi^{2} / 9+\pi^{2}\right) x=F_{N}(x),
$$

where the linear part is no more resonant with respect to given boundary conditions, $F_{N}$ is bounded and given by

$$
F_{N}(x)=\left\{\begin{array}{rc}
-0.002 \pi^{32}, & x>1.07534 \cdot 10^{15}, \\
\pi^{2} x-|x|^{\frac{16}{15}} \operatorname{sgn} x, & -1.07534 \cdot 10^{15} \leq x \leq 1.07534 \cdot 10^{15}, \\
0.002 \pi^{32}, & x<-1.07534 \cdot 10^{15} .
\end{array}\right.
$$

The function $F(x)$ is continuous and bounded by the number $M$. Therefore the quasilinear BVP $(5.14)$ has a solution $x(t)$, which satisfies the integral form

$$
x(t)=\int_{0}^{1} G(t, s) F_{N}(x(s)) d s,
$$

where $G$ is the Green's function for the problem

$$
x^{\prime \prime}+\frac{13 \pi^{2}}{9} x=0, \quad x(0)=0, \quad x(1)=x\left(\frac{1}{2}\right) .
$$


It follows from (5.15) that

$$
|x(t)| \leq \Gamma M \quad \forall t \in[0,1],
$$

where $\Gamma=3\left(1+\left|\sin \frac{\sqrt{13} \pi}{6}\right|\right) /\left(2 \sqrt{13} \pi\left|\sin \frac{\sqrt{13} \pi}{12}\right|\left|\cos \frac{\sqrt{13} \pi}{4}\right|\right)$, holds.

Since

$$
\Gamma M=\frac{3\left(1+\left|\sin \frac{\sqrt{13} \pi}{6}\right|\right)}{2 \sqrt{13} \pi\left|\sin \frac{\sqrt{13} \pi}{12}\right|\left|\cos \frac{\sqrt{13} \pi}{4}\right|} \cdot 0.002 \pi^{32} \approx 6.442 \cdot 10^{13}<1.075 \cdot 10^{15} \approx N,
$$

then the solution $x(t)$ satisfies inequality

$$
|x(t)| \leq N \quad \forall t \in[0,1] .
$$

This means that the BVP (5.13) allows for quasilinearization for $\varepsilon=\pi$ and the resulting coefficient $\sqrt{4 \pi^{2} / 9+\varepsilon^{2}}=\sqrt{13} \pi / 3$ lies in the nonresonance interval $(2 \pi / 3,2 \pi)$, and the solution $x(t)$ of the quasilinear BVP (5.14) is also a solution of the original resonant BVP (5.13).

The BVP (5.13) also allows for quasilinearization for $\varepsilon=8 \pi / 3$ and the resulting coefficient $\sqrt{4 \pi^{2} / 9+\varepsilon^{2}}=2 \sqrt{17} \pi / 3$ lies in the nonresonance interval $(2 \pi, 10 \pi / 3)$; and for $\varepsilon=11 \pi / 3$, when the resulting coefficient $\sqrt{4 \pi^{2} / 9+\varepsilon^{2}}=$ $2 \sqrt{17} \pi / 3$ lies in the nonresonance interval $(10 \pi / 3,4 \pi)$.

Applying the shooting method we find different solutions for each quasilinearization. At the first quasilinearization we find the trivial solution and solutions on the next two quasilinearizations are depicted in Figure 2.

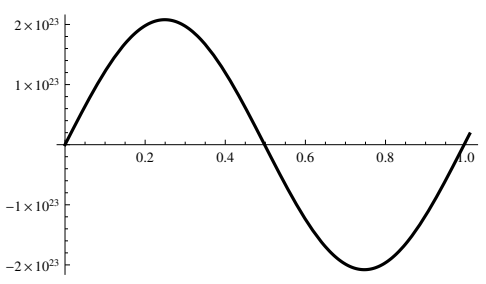

(a) Solution of the BVP (5.13) given for the initial data $x(0)=0$, $x^{\prime}(0)=13 \cdot 10^{23}$. Solution related to quasilinearization with linear part $\left(L_{2} x\right)(t):=x^{\prime \prime}+\frac{68 \pi^{2}}{9} x$ of the BVP (5.13).

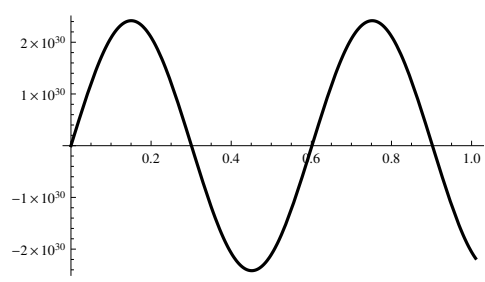

(b) Solution of the BVP (5.13) given for the initial data $x(0)=0$, $x^{\prime}(0)=25 \cdot 10^{30}$. Solution related to quasilinearization with linear part $\left(L_{2} x\right)(t):=x^{\prime \prime}+\frac{125 \pi^{2}}{9} x$ of the BVP (5.13).

Figure 2. Different solutions of the BVP (5.13).

\section{Conclusions}

We showed that the three-point BVP can be studied by using a quasilinearization process. 
Two examples of the Emden-Fowler type BVP are considered in detail showing the quasilinearization approach in action.

By using quasilinearization process with different linear parts we can state the existence of different solutions of a problem thus obtaining multiplicity of results.

\section{References}

[1] W. Feng and J.R.L. Webb. Solvability of a three point nonlinear boundary value problem at resonance. Nonlinear Analysis TMA, 30(6):3227-3238, 1997. http://dx.doi.org/10.1016/S0362-546X(96)00118-6.

[2] W. Feng and J.R.L. Webb. Solvability of $m$-point boundary value problem with nonlinear growth. J. Math. Anal. Appl., 212(2):467-480, 1997. http://dx.doi.org/10.1006/jmaa.1997.5520.

[3] C.P. Gupta. Solvability of a three-point nonlinear boundary value problem for second order ordinary differential equation. Journal of mathematical analysis and applications, 168(2):540-551, 1992. http://dx.doi.org/10.1016/0022247X(92)90179-H.

[4] C.P. Gupta. Solvability of a multi-point boundary value problem at resonance. Results Math., 28(3):270-276, 1995. http://dx.doi.org/10.1007/BF03322257.

[5] C.P. Gupta. A Dirichlet type multi-point boundary value problem for second order ordinary differential equations. Nonlinear Anal., 26(5):925-931, 1996. http://dx.doi.org/10.1016/0362-546X(94)00338-X.

[6] C.P. Gupta, S.K. Ntouyas and P.Ch. Tsamatos. On the solvability of some multi-point boundary value problems. Appl. Math., 41(1):1-17, 1996.

[7] V. Il'in and E. Moiseev. Nonlocal boundary value problem of the first kind for a Sturm-Liouville operator in its differential and finite difference aspects. Differential Equations, 23:803-810, 1987.

[8] V. Il'in and E. Moiseev. Nonlocal boundary value problem of the second kind for a Sturm-Liouville operator. Differential Equations, 23:979-987, 1987.

[9] B. Liu. Positive solutions of a nonlinear three-point boundary value problem. Computers and Mathematics with Applications, 44(1-2):201-211, 2002. http://dx.doi.org/10.1016/S0898-1221(02)00141-4.

[10] R. Ma. Existence theorems for a second order $m$-point boundary value problem. Journal of mathematical analysis and applications, 211(2):545-555, 1997. http://dx.doi.org/10.1006/jmaa.1997.5416.

[11] R. Ma. Positive solutions for a nonlinear three-point boundary value problem. Electron. J. Diff. Eqns., 34:1-8, 1999.

[12] R. Ma. A survey on nonlocal boundary value problem. Applied Mathematics E-Notes, 7:257-279, 2007.

[13] M. Moshinsky. Sobre los problemas de condiciones a la frontiera en una dimension de caracteristicas discontinuas. Bol. Soc. Mat. Mexicana, 7:1-25, 1950.

[14] S.K. Ntouyas. Nonlocal initial and boundary value problems: a survey. In A. Cañada, P. Drábek and A. Fonda(Eds.), Handbook of Differential Equations, volume 2 of Ordinary Differential Equations, pp. 461-558, North Holland, 2005. Elsevier. 
[15] B. Przeradzki and R. Sta'nczy. Solvability of a multi-point boundary value problem at resonance. Journal of Mathematical Analysis and Applications, 264:253261, 2001.

[16] S. Roman and A. Štikonas. Green's functions for stationary problems with nonlocal boundary conditions. Lith. Math. J., 49(2):190-202, 2009. http://dx.doi.org/10.1007/s10986-009-9041-0.

[17] Y. Sun and L. Liu. Solvability for a nonlinear second-order three-point boundary value problem. J. Math. Anal. Appl., 296(1):265-275, 2004. http://dx.doi.org/10.1016/j.jmaa.2004.04.013.

[18] N. Sveikate. Quasilinearization for resonant nonlinear boundary value problems with neumann boundary conditions. Proceedings of IMCS of University of Latvia, 13:110-119, 2013.

[19] N. Sveikate. Resonant problems by quasilinearization. International Journal of Differential Equations, 2014:8 pages, 2014. http://dx.doi.org/10.1155/2014/564914.

[20] N. Sveikate and F. Sadyrbaev. Quasilinearization for resonant boundary-value problems with mixed boundary conditions. Nonlinear Oscillations, 17(1):112126, 2014.

[21] N.I. Vasilyev and Yu.A. Klokov. Foundations of the Theory of Boundary Value Problems for Ordinary Differential Equations. Zinatne, Riga, 1978 (Russian).

[22] A.K. Verma and M. Singh. A note on existence results for a class of three-point nonlinear BVPs. Math. Model. Anal., 20(4):457-470, 2015. http://dx.doi.org/10.3846/13926292.2015.1065293.

[23] J.R.L. Webb. A unified approach to nonlocal boundary value problems. Dynamic Systems and Applications, 5:510-515, 2008.

[24] J.R.L. Webb and G. Infante. Positive solutions of nonlocal boundary value problems: a unified approach. Journal of the London Mathematical Society, 74(3):673-693, 2006. http://dx.doi.org/10.1112/S0024610706023179.

[25] J.R.L. Webb and G. Infante. Nonlocal boundary value problems of arbitrary order. Journal of the London Mathematical Society, 79(1):238-258, 2009. http://dx.doi.org/10.1112/jlms/jdn066.

[26] I. Yermachenko and F. Sadyrbaev. Quasilinearization and multiple solutions of the emden-folwer type equation. Math. Model. Anal., 10:41-50, 2005. http://dx.doi.org/10.1080/13926292.2005.9637269.

[27] I. Yermachenko and F. Sadyrbaev. Types of solutions and multiplicity results for two-point nonlinear boundary value problems. Nonlinear Analysis, 63(1):e1725e1735, 2005. 